\title{
Ergodic Theorem in Grand Variable Exponent Lebesgue Spaces
}

\author{
Cihan Unal*
}

\begin{abstract}
We consider several fundamental properties of grand variable exponent Lebesgue spaces. Moreover, we discuss Ergodic theorems in these spaces whenever the exponent is invariant under the transformation.
\end{abstract}

Keywords: Variable exponent grand Lebesgue space; Ergodic theorem; probability measure.

AMS Subject Classification (2020): Primary: 28D05 ; Secondary: 43A15; 46E30.

${ }^{*}$ Corresponding author

\section{Introduction}

In 1992, Iwaniec and Sbordone [14] introduced grand Lebesgue spaces $L^{p)}(\Omega),(1<p<\infty)$, on bounded sets $\Omega \subset \mathbb{R}^{d}$ with applications to differential equations. A generalized version $L^{p), \theta}(\Omega)$ appeared in Greco et al. [13]. These spaces has been intensively investigated recently due to several applications, see $[2,5,9,11,15,18]$. Also the solutions of some nonlinear differential equations were studied in these spaces, see $[10,13]$. The variable exponent Lebesgue spaces (or generalized Lebesgue spaces) $L^{p(.)}$ appeared in literature for the first time in 1931 with an article written by Orlicz [17]. Kováčik and Rákosník [16] introduced the variable exponent Lebesgue space $L^{p(.)}\left(\mathbb{R}^{d}\right)$ and Sobolev space $W^{k, p(.)}\left(\mathbb{R}^{d}\right)$ in higher dimensions Euclidean spaces. The spaces $L^{p(.)}\left(\mathbb{R}^{d}\right)$ and $L^{p}\left(\mathbb{R}^{d}\right)$ have many common properties such as Banach space, reflexivity, separability, uniform convexity, Hölder inequalities and embeddings. A crucial difference between $L^{p(.)}\left(\mathbb{R}^{d}\right)$ and $L^{p}\left(\mathbb{R}^{d}\right)$ is that the variable exponent Lebesgue space is not invariant under translation in general, see [6, Lemma 2.3] and [16, Example 2.9]. For more information, we refer $[3,7,8]$. Moreover, the space $L^{p(.)}(\Omega)$ was studied by [1], where $\Omega$ is a probability space. The grand variable exponent Lebesgue space $L^{p(.), \theta}(\Omega)$ was introduced and studied by Kokilashvili and Meskhi [15]. In this work, they established the boundedness of maximal and Calderon operators in these spaces. Moreover, the space $L^{p(\cdot), \theta}(\Omega)$ is not reflexive, separable, rearrangement invariant and translation invariant.

In this study, we give some basic properties of $L^{p(.), \theta}(\Omega)$, and consider Birkhoff's Ergodic Theorem in the context of a certain subspace of the grand variable exponent Lebesgue space $L^{p(.), \theta}(\Omega)$. So, we have more general results in sense to Gorka [12] in these spaces.

\section{Notations and Preliminaries}

Definition 2.1. Assume that $(\Omega, \Sigma, \mu)$ is a probability space, that is, $\Sigma$ is a $\sigma$-algebra and $\mu$ is a measure on $\Sigma$ satisfying $\mu(\Omega)=1$. Let $p():. \Omega \longrightarrow[1, \infty)$ be a measurable function (variable exponent) such that

$$
1 \leq p^{-}=\underset{x \in \Omega}{\operatorname{essinf}} p(x) \leq \underset{x \in \Omega}{\operatorname{esssup}} p(x)=p^{+}<\infty .
$$

Received : 31-01-2020, Accepted : 05-07-2020 
The variable exponent Lebesgue space $L^{p(.)}(\Omega)$ is defined as the set of all measurable functions $f$ on $\Omega$ such that $\varrho_{p(.)}(\lambda f)<\infty$ for some $\lambda>0$, equipped with the Luxemburg norm

$$
\|f\|_{p(.)}=\inf \left\{\lambda>0: \varrho_{p(.)}\left(\frac{f}{\lambda}\right) \leq 1\right\}
$$

where $\varrho_{p(.)}(f)=\int_{\Omega}|f(x)|^{p(x)} d \mu(x)$. The space $L^{p(.)}(\Omega)$ is a Banach space with respect to $\|\cdot\|_{p(.)}$. Moreover, the norm $\|.\|_{p(.)}$ coincides with the usual Lebesgue norm $\|\cdot\|_{p}$ whenever $p()=$.$p is a constant function. Let p^{+}<\infty$. Then $f \in L^{p(.)}(\Omega)$ if and only if $\varrho_{p(.)}(f)<\infty$, see [16].

Definition 2.2. Let $\theta>0$. The grand variable exponent Lebesgue spaces $L^{p(.), \theta}(\Omega)$ is the class of all measurable functions for which

$$
\|f\|_{p(.), \theta}=\sup _{0<\varepsilon<p^{-}-1} \varepsilon^{\frac{\theta}{p^{-}-\varepsilon}}\|f\|_{p(.)-\varepsilon}<\infty .
$$

When $p()=$.$p is a constant function, these spaces coincide with the grand Lebesgue spaces L^{p), \theta}(\Omega)$.

It is easy to see that we have

$$
L^{p(.)} \hookrightarrow L^{p(\cdot), \theta} \hookrightarrow L^{p(.)-\varepsilon} \hookrightarrow L^{1}, 0<\varepsilon<p^{-}-1
$$

due to $|\Omega|<\infty$, see $[4,15,18]$.

Remark 2.1. Let $C_{0}^{\infty}(\Omega)$ be the space of smooth functions with compact support in $\Omega$. It is well known that $C_{0}^{\infty}(\Omega)$ is not dense in $L^{p(.), \theta}(\Omega)$, i.e., the closure of $C_{0}^{\infty}(\Omega)$ with respect to the $\|\cdot\|_{p(.), \theta}$ norm does not coincide with the space $L^{p(.), \theta}(\Omega)$. Now, we denote $\left[L^{p(.)}(\Omega)\right]_{p(.), \theta}$ as the closure of $C_{0}^{\infty}(\Omega)$ in $L^{p(.), \theta}(\Omega)$. Hence this closure is obtained as

$$
\left\{f \in L^{p(.), \theta}(\Omega): \lim _{\varepsilon \rightarrow 0} \varepsilon^{\frac{\theta}{p^{-}-\varepsilon}}\|f\|_{p(.)-\varepsilon}=0\right\}
$$

, see $[4,13,15]$. Moreover, we have

$$
C_{0}^{\infty}(\Omega) \subset L^{p(.)}(\Omega) \subset\left[L^{p(.)}(\Omega)\right]_{p(.), \theta} \text { and }\left[L^{p(.)}(\Omega)\right]_{p(.), \theta}=\overline{C_{0}^{\infty}(\Omega)} .
$$

Definition 2.3. Let $(G, \Sigma, \mu)$ be a measure space. A measurable function $T: G \longrightarrow G$ is called a measure-preserving transformation if

$$
\mu\left(T^{-1}(A)\right)=\mu(A)
$$

for all $A \in \Sigma$.

\section{Main Results}

In the following theorem, we obtain more general result than $\left[12\right.$, Theorem 3.1] since $L^{p(.)}(\Omega) \subset\left[L^{p(.)}(\Omega)\right]_{p(.), \theta} \subset$ $L^{p(.), \theta}(\Omega)$.

Theorem 3.1. Let $(\Omega, \Sigma, \mu)$ be a probability space and $T: \Omega \longrightarrow \Omega$ a measure preserving transformation. Moreover, if $p($.$) is$ T-invariant, i.e., $p(T())=.p($.$) , then$

(i) The limit

$$
f_{a v}(x)=\lim _{n \rightarrow \infty} \frac{1}{n} \sum_{j=0}^{n-1} f\left(T^{j}(x)\right)
$$

exists for all $f \in L^{p(.), \theta}(\Omega)$ and almost each point $x \in \Omega$, and $f_{a v} \in L^{p(.), \theta}(\Omega)$.

(ii) For every $f \in L^{p(.), \theta}(\Omega)$, we have

$$
\begin{gathered}
f_{a v}(x)=f_{a v}(T(x)), \\
\int_{\Omega} f_{a v} d \mu=\int_{\Omega} f d \mu .
\end{gathered}
$$


(iii) For all $f \in\left[L^{p(.)}(\Omega)\right]_{p(.), \theta^{\prime}}$ we get

$$
\lim _{n \rightarrow \infty}\left\|f_{a v}-\frac{1}{n} \sum_{j=0}^{n-1} f \circ T^{j}\right\|_{p(.), \theta}=0
$$

Proof. By (2.1), the existence of limit $f_{a v}(x)$ for almost every point in $\Omega$ follows from the standard Birkhoof's Theorem, see [12]. By Fatou's Lemma and the definition of the norm $\|.\|_{p(.), \theta}$, we have

$$
\begin{aligned}
\int_{\Omega}\left|f_{a v}(x)\right|^{p(x)-\varepsilon} d \mu & =\int_{\Omega}\left|\lim _{n \rightarrow \infty} \frac{1}{n} \sum_{j=0}^{n-1} f\left(T^{j}(x)\right)\right|^{p(x)-\varepsilon} d \mu \\
& \leq \int_{\Omega} \lim _{n \rightarrow \infty}\left(\frac{1}{n} \sum_{j=0}^{n-1}\left|f\left(T^{j}(x)\right)\right|\right)^{p(x)-\varepsilon} d \mu \\
& \leq \liminf _{n \rightarrow \infty} \int_{\Omega}\left(\frac{1}{n} \sum_{j=0}^{n-1}\left|f\left(T^{j}(x)\right)\right|\right)^{p(x)-\varepsilon} d \mu \\
& \leq \liminf _{n \rightarrow \infty} \frac{1}{n} \sum_{j=0}^{n-1} \int_{\Omega}\left|f\left(T^{j}(x)\right)\right|^{p(x)-\varepsilon} d \mu
\end{aligned}
$$

for any $\varepsilon \in\left(0, p^{-}-1\right)$. Here, we used convexity and Jensen inequality in last step. Moreover, since $T$ is a measure preserving map and $p($.$) is T$-invariant, we get

$$
\int_{\Omega}|f(T(x))|^{p(x)-\varepsilon} d \mu=\int_{\Omega}|f(T(x))|^{p(T(x))-\varepsilon} d \mu=\int_{\Omega}|f(x)|^{p(x)-\varepsilon} d \mu .
$$

It follows that

$$
\int_{\Omega}\left|f_{a v}(x)\right|^{p(x)-\varepsilon} d \mu \leq \int_{\Omega}|f(x)|^{p(x)-\varepsilon} d \mu<\infty .
$$

Thus, we obtain

$$
\begin{aligned}
\left\|f_{a v}\right\|_{p(.), \theta} & =\sup _{0<\varepsilon<p^{-}-1} \varepsilon^{\frac{\theta}{p^{-}-\varepsilon}}\left\|f_{a v}\right\|_{p(.)-\varepsilon} \\
& \leq \sup _{0<\varepsilon<p^{-}-1} \varepsilon^{\frac{\theta}{p^{-}-\varepsilon}}\|f\|_{p(.)-\varepsilon}<\infty
\end{aligned}
$$

and $f_{a v} \in L^{p(.), \theta}(\Omega)$. This completes (i). By the Ergodic Theorem in the classical Lebesgue spaces (see [12]), we have (3.1) and (3.2) immediately. In order to prove (3.3), we assume that $f \in C_{0}^{\infty}(\Omega)$. Thus, $f \in L^{\infty}(\Omega)$ and

$$
\begin{aligned}
\lim _{n \rightarrow \infty}\left|f_{a v}(x)-\frac{1}{n} \sum_{j=0}^{n-1} f\left(T^{j}(x)\right)\right|^{p(x)-\varepsilon} & =0, \text { a.e. } \\
\left\|f_{a v}\right\|_{L^{\infty}(\Omega)} & \leq\|f\|_{L^{\infty}(\Omega)}
\end{aligned}
$$

for any $\varepsilon \in\left(0, p^{-}-1\right)$. Therefore, we have

$$
\begin{aligned}
\left|f_{a v}(x)-\frac{1}{n} \sum_{j=0}^{n-1} f\left(T^{j}(x)\right)\right|^{p(x)-\varepsilon} & \leq\left|\|f\|_{L^{\infty}(\Omega)}+\frac{1}{n} \sum_{j=0}^{n-1}\left\|f\left(T^{j}\right)\right\|_{L^{\infty}(\Omega)}\right|^{p(x)-\varepsilon} \\
& \leq 2^{p^{+}}\left(\|f\|_{L^{\infty}(G)}+1\right)^{p^{+}-\varepsilon} \in L^{1}(\Omega) .
\end{aligned}
$$


Hence, by Lebesgue dominated convergence theorem (see [7]), we have (3.3) and provided $f \in C_{0}^{\infty}(\Omega)$. Since $C_{0}^{\infty}(\Omega)$ is dense in $\left[L^{p(.)}(\Omega)\right]_{p(.), \theta}$ with respect to the norm $\|.\|_{p(.), \theta}$, for any $f \in\left[L^{p(.)}(\Omega)\right]_{p(.), \theta}$ and $\eta>0$ there is a $g \in C_{0}^{\infty}(\Omega)$ such that

$$
\|f-g\|_{p(.), \theta}<\eta \text {. }
$$

By the previous step, there is an $n_{0}$ such that

$$
\left\|g_{a v}-\frac{1}{n} \sum_{j=0}^{n-1} g \circ T^{j}\right\|_{p(.)-\varepsilon}<\eta
$$

for $n \geq n_{0}$ and $\varepsilon \in\left(0, p^{-}-1\right)$. Hence, we have

$$
\left\|g_{a v}-\frac{1}{n} \sum_{j=0}^{n-1} g \circ T^{j}\right\|_{p(.), \theta}<\eta
$$

by (3.6) and the definition of the norm $\|\cdot\|_{p(.), \theta}$. This follows from (3.4), (3.5) and (3.7) that

$$
\begin{aligned}
\left\|f_{a v}-\frac{1}{n} \sum_{j=0}^{n-1} f \circ T^{j}\right\|_{p(.), \theta} \leq & \left\|f_{a v}-g_{a v}\right\|_{p(.), \theta}+\left\|g_{a v}-\frac{1}{n} \sum_{j=0}^{n-1} g \circ T^{j}\right\|_{p(.), \theta} \\
& +\left\|\frac{1}{n} \sum_{j=0}^{n-1}(f-g) \circ T^{j}\right\|_{p(.), \theta} \\
\leq & 2\|f-g\|_{p(.), \theta}+\left\|g_{a v}-\frac{1}{n} \sum_{j=0}^{n-1} g \circ T^{j}\right\|_{p(.), \theta} \\
& <\frac{\eta}{2}+\frac{\eta}{2}=\eta .
\end{aligned}
$$

That is the desired result.

\section{References}

[1] Aoyama, H.: Lebesgue spaces with variable exponent on a probability space. Hiroshima Math. J. 39, 207-216 (2009). https://doi.org/10.32917/hmj/1249046337

[2] Capone, C., Formica, M. R., Giova, R.: Grand Lebesgue spaces with respect to measurable functions. Nonlinear Anal. 85, 125-131 (2013). https://doi.org/10.1016/j.na.2013.02.021

[3] Cruz-Uribe, D. V., Fiorenza, A.: Variable Lebesgue Spaces Foundations and Harmonic Analysis. Springer, New York (2013).

[4] Danelia, N., Kokilashvili, V.: Approximation by trigonometric polynomials in the framework of variable exponent grand Lebesgue spaces. Georgian Math. J. 23 (1), 43-53 (2016). https://doi.org/10.1515/gmj-2015-0059

[5] Di Fratta, G., Fiorenza, A.: A direct approach to the duality of grand and small Lebesgue spaces. Nonlinear Anal. 70 (7), 2582-2592 (2009). https:/ / doi.org/10.1016/j.na.2008.03.044

[6] Diening, L.: Maximal function on generalized Lebesgue spaces $L^{p(\cdot)}$. Mathematical Inequalities and Applications 7, 245-253 (2004). https://doi.org/10.7153/mia-07-27

[7] Diening, L., Harjulehto, P., Hästö, P., Růžička, M.: Lebesgue and Sobolev Spaces with Variable Exponents. Springer-Verlag, Berlin (2011).

[8] Fan, X. L., Zhao, D.: On the spaces $L^{p(x)}(\Omega)$ and $W^{k, p(x)}(\Omega)$. J. Math. Anal. Appl. 263 (2), $424-446$ (2001). https://doi.org/10.1006/jmaa.2000.7617 
[9] Fiorenza, A.: Duality and reflexivity in grand Lebesgue spaces. Collect. Math. 51 (2), 131-148 (2000).

[10] Fiorenza, A., Sbordone, C.: Existence and uniqueness results for solutions of nonlinear equations with right hand side in $L^{1}$. Studia Math. 127 (3), 223-231 (1998).

[11] Fiorenza, A., Gupta, B., Jain, P.: The maximal theorem in weighted grand Lebesgue spaces. Stud. Math. 188 (2), 123-133 (2008). https:/ / doi.org/10.4064/sm188-2-2

[12] Gorka, P.: Ergodic theorem in variable Lebesgue spaces. Period Math. Hung. 72, $243-247$ (2016). https://doi.org/10.1007/s10998-016-0125-4

[13] Greco, L., Iwaniec, T., Sbordone, C.: Inverting the p-harmonic operator. Manuscripta Math. 92, 249-258 (1997). https://doi.org/10.1007/BF02678192

[14] Iwaniec, T., Sbordone, C.: On integrability of the Jacobien under minimal hypotheses. Arch. Rational Mechanics Anal. 119, 129-143 (1992). https:/ / doi.org/10.1007/BF00375119

[15] Kokilashvili, V., Meskhi, A.: Maximal and Calderon -Zygmund operators in grand variable exponent Lebesgue spaces. Georgian Math. J. 21, 447-461 (2014). https:/ / doi.org/10.1515/gmj-2014-0047

[16] Kováčik, O., Rákosník, J.: On spaces $L^{p(x)}$ and $W^{k, p(x)}$. Czechoslovak Math. J. 41(116) (4), 592-618 (1991).

[17] Orlicz, W.: Über Konjugierte Exponentenfolgen. Studia Math. 3, 200-212 (1931). https://doi.org/10.4064/sm-3-1200-211

[18] Rafeiro, H., Vargas, A.: On the compactness in grand spaces, Georgian Math. 22 (1), 141-152 (2015). https://doi.org/10.1515/gmj-2014-0060

\section{Affiliations}

CiHAN UnAL

AdDress: Assessment, Selection and Placement Center, 06800, Ankara, Turkey.

E-MAIL: cihanunal88@gmail.com

ORCID ID:0000-0002-7242-393X 